\title{
AVALIAÇÃo dA CONDIÇÃO HIGIÊNICO SANITÁRIA NO PREPARO DE MERENDA ESCOLAR NAS ESCOLAS DA REDE PÚBLICA DA CIDADE DE RIANÁPOLIS-GO.
}

\author{
EVALUTION OF THE HYGIENIC AND SANITARY CONDITION IN THE \\ PREPARATION OF THE SCHOOL LUNCH ON PUBLIC SCHOOLS IN THE \\ CITY OF RIANÁPOLIS STATE OF GOIÁS.
}

\section{Milce Costa}

Docente do curso de Farmácia da FACER- Unidade Ceres. Doutora em Medicina Tropical UFG. milcebiomol@yahoo.com.br Av. Brasil, S/N, Qd. 13 Morada Verde Ceres - Go. CEP: 76.300-000.

\section{Gilmar Aires da Silva}

Docente do curso de Farmácia da FACER- Unidade Ceres. Mestre em Química UFG. gilmaraires@hotmail.com Av. Brasil, S/N, Qd. 13 Morada Verde Ceres - Go. CEP: 76.300000 .

\section{Karla Cássia Nunes}

Acadêmica do Curso de Farmácia da FACER- Faculdade de Ceres-GO: karlanuneshta@hotmail.com

\section{Karlenny Kristianny Gomides Silva}

Acadêmica do Curso de Farmácia da FACER- Faculdade de Ceres-GO: karlennyfarmacia@hotmail.com

\section{RESUMO}

Introdução: o processo de preparo da merenda escolar exige o cumprimento incondicional de todas as medidas sanitárias estabelecidas pela Agência Nacional de Vigilância Sanitária ANVISA. Objetivo: O objetivo desde estudo foi avaliar as condições higiênico-sanitárias no preparo dos alimentos das escolas públicas da cidade de Rianápolis-GO. Metodologia: Realizou-se um estudo observacional das cozinhas das três escolas através da aplicação de check-list baseado nas RDCs 275 de 21/10/2002 e 216 de 15/09/2004. Resultados e discussão: Os resultados do check-list foram mostrados através de conformidades e não conformidades de acordo com a legislação vigente. A escola A, apresentou 17 (68\%) de 
conformidade; a escola B, 18 (72\%) de conformidade e a escola C, $16(64 \%)$ de conformidade. A avaliação do check-list neste estudo mostrou-se satisfatória, apesar da constatação de algumas não-conformidades. Tal resultado pode ser entendido como um indício da aplicação das RDCs vigentes no país. Conclusão: A maioria dos casos de DTAs poderiam ser prevenidas se os manipuladores fossem treinados e preparados dentro dos princípios das boas práticas no preparo de alimentos, havendo sempre uma parceria com a vigilância epidemiológica por meio de exames parasitológicos para que todos esses indivíduos possam se assegurar de alimentos com qualidade.

Palavras-chave: Manipuladores de alimentos. DTAs. Check-list. Condições higiênicosanitárias.

\begin{abstract}
Introduction: The process of preparation of school meals requires the achievement of all sanitary measures established by the Agência Nacional de Vigilância Sanitária - ANVISA. Goal: This study's goal was to evaluate the hygienic sanitary conditions in the preparation of food in public school in the city of Rianápolis state of Goiás. Methodology: An observational study was held in the kitchens of the three schools the application of check-list based on the RDCs 275 from 10/21/2002 and 216 from 09/15/2004. Results and Discussion: The results of the check-list were shown through conformities and non conformities accordingly to the legislation. The school A, presented 17 (68\%) of conformity: The school B, $18(72 \%)$ of conformity, and the school C $16(64 \%)$ of conformity. The check-list evaluation in this study was satisfactory, in spite of the finding of some non- conformities. This result can be understood as an evidence of the applications of the RDCs from the country. Conclusion: Most of the cases of FBDs could be prevented if the handlers were prepared into the good practices principles and in the food preparation, always keeping a partnership with the epidemiological surveillance, via parasitological tests to ensure these individuals eat quality food.
\end{abstract}

Key words: Food handles. FBDs. Check-list. Hygienic sanitary conditions.

\title{
Endereço para correspondência
}

Av. Brasil, S/N, Qd. 13 Morada Verde Ceres - Go. CEP: 76.300-000. milcebiomol@yahoo.com.br 


\section{INTRODUÇÃO}

Há muito se reconheceu que a questão alimentar é também responsabilidade do Estado que passou a reconhecer o direito à alimentação como uma de suas funções. No caso específico do Brasil, a ênfase foi dada a alimentação escolar que desde os anos 1940 vem sendo abordada através de programas estatais sendo o mais importante o Programa Nacional de Alimentação Escolar - PNAE, que tem como objetivo atender os alunos do primeiro grau matriculados nas redes públicas e filantrópicas. E esta alimentação deve atender às especificações nutricionais pré-determinadas pelo programa (FNDE, 2006).

Deve-se ainda salientar que o processo de preparo da merenda escolar exige o cumprimento incondicional de todas as medidas sanitárias estabelecidas pela Agência Nacional de Vigilância Sanitária - ANVISA (ALMEIDA; SOUZA, 2007). Neste sentido, pode-se destacar a Resolução - RDC ANVISA nº. 216/04 que foi elaborada para proteger a saúde da população contra doenças provocadas pelo consumo de alimentos contaminados. Essa Resolução estabelece normas específicas de boas práticas para serviços de alimentação (BRASIL, 2004).

As doenças transmitidas por alimentos (DTAs) são um dos principais fatores que colaboram para os índices de morbidade nos países da América Latina, sendo assim a qualidade higiênico-sanitária como fator de segurança alimentar vem sendo estudada e vastamente discutida (AKUTSU et al., 2005).

As DTAs são enfermidades causadas através da ingestão de água ou alimentos contaminados por diversos grupos de micro-organismos, incluindo bactérias, fungos, protozoários, vírus, substâncias danosas e partículas que não estavam presentes originalmente nestes alimentos. Seus sintomas são caracterizados por um conjunto de perturbações gástricas, envolvendo geralmente vômitos, diarréia, febres e dores abdominais, que podem ocorrer individualmente ou em combinação (PINTO, 1996). Geralmente tais interferentes são introduzidos nos alimentos pelos manipuladores ou pelo meio ambiente e outros ocorrem acidentalmente quando não são adotadas as boas práticas de higiene. Portanto, garantir um alimento inócuo que não ofereça risco ao consumidor é sempre um desafio (ROUGEMONT, 2007).

Estudos feitos no Brasil referentes ao período compreendido entre 1999 a 2011 mostram que foram notificados ao Ministério da Saúde 8.663 surtos de DTA, com o acometimento de 163.425 pessoas e registro de 112 óbitos. Entre os anos de 2000 a 2011 a maior incidência de surtos apontam para o encontro de Salmonella spp, seguida de S. aureus, E.coli, B.cereus entre outros (BRASIL, 2011). 
Neste contexto, as DTAs constituem uma das principais preocupações relacionadas à saúde pública, tendo uma importância ainda maior no caso de alimentos produzidos em escolas, uma vez que os consumidores desses alimentos são crianças e adolescentes, sendo este um grupo mais susceptível a toxinfecções alimentares (DA SILVA; FORTUNA, 2011).

É importante salientar que os manipuladores em muitos casos não estão cientes dos riscos que expõe a população ao manusearem os alimentos de maneira inadequada (MELLO, 2009). A maioria dos casos de doenças de origem alimentar poderia ser prevenida se estes trabalhadores fossem melhor treinados e preparados dentro dos princípios das boas práticas no preparo de alimentos (PANIZZA et al., 2011). Portanto, os manipuladores de alimentos devem entender as boas práticas de higiene como uma forma de proteger a sua saúde e a dos consumidores, uma vez que eles são a principal via de contaminação dos alimentos (MELLO, 2009).

Segundo o Ministério da Saúde as residências são os locais com maior ocorrência de surtos de DTAs $(48,5 \%)$, seguidas de restaurantes (18,8\%) e escolas (11,6\%). Sabe-se ainda que a maioria dessas doenças está ligada aos precários hábitos de higiene pessoal dos próprios manipuladores e da deficiente higienização do ambiente de preparo (BRASIL, 2005).

Assim, considerando que os manipuladores de merenda escolar podem ser potenciais transmissores de DTAs em virtude de procedimentos deficientes de higienização, o presente estudo se propõe a avaliar as condições físicas e higiênico sanitárias das cozinhas e dos manipuladores de alimentos responsáveis pela merenda nas escolas públicas da cidade de Rianápolis- GO.

\section{METODOLOGIA}

Realizou-se um estudo observacional das condições higiênico sanitárias no preparo de merenda escolar entre os meses de setembro a outubro de 2012 nas cozinhas de três escolas da rede pública de ensino do município de Rianápolis-GO, sendo duas da Rede Estadual e outra da Rede Municipal.

\section{Avaliação das condições higiênico-sanitárias}


A verificação das condições higiênico-sanitárias das cozinhas das escolas incluídas nesta pesquisa foi desenvolvida através de um estudo observacional realizado através de aplicação de check-list baseado nas RDCs 275 de 21/10/2002 e 216 de 15/09/2004. O check-list utilizado contemplou 25 itens (Apêndice 1) e abordou as condições físicas e higiênico-sanitárias das cozinhas das escolas e dos manipuladores de alimentos. Através de sua aplicação registrou-se as conformidades e não conformidades constatadas nas cozinhas pesquisadas segundo a legislação vigente. $\mathrm{O}$ critério utilizado para aplicação do check-list estabelecido em nosso estudo baseou-se na marcação de não conformidade quando verificouse pelo menos um item em discordância dos estabelecidos nas RDCs 216/2004 e 275/2002. A aplicação do check-list ocorreu em um único dia em todas as escolas, e sem aviso prévio para que a rotina de trabalho não fosse alterada.

\section{Análise dos dados}

As informações obtidas foram registradas e calculou-se a porcentagem das respostas que se apresentavam em conformidade ou não com os padrões preconizados de higiene pessoal e Boas Práticas da legislação vigente. As informações obtidas após a realização do check-list foram apresentadas na forma de tabelas.

\section{RESULTADOS E DISCUSSÃO}

Neste estudo foram avaliadas três escolas da rede pública de Rianápolis-GO através da aplicação de um check-list que avaliou as condições físicas e higiênico-sanitárias das cozinhas e dos manipuladores de alimentos. Os resultados do check-list foram mostrados através de conformidades e não conformidades de acordo com as RDC 216/2004 e 275/2002 (Tabela 1, 2, 3). A escola A, apresentou 17 (68\%) de conformidade e 8 (32\%) de não conformidades; a escola B, $18(72 \%)$ conformidade e $7(28 \%)$ de não conformidades e a escola C, 16 (64\%) de conformidade e 9 (36\%) de não conformidades. 
Tabela 1- Verificação de conformidades e não conformidades das condições físicas e higiênico-sanitárias das cozinhas e dos manipuladores encontradas na escola "A" após aplicação de check-list* $^{*}$.

\section{Escola A}

$\mathrm{N}^{*} \quad \%$

Conformidades

$17 \quad 68$

Não conformidades

$8 \quad 32$

\begin{tabular}{lll}
\hline Total & 25 & 100
\end{tabular}

$N^{*}$ - Numero de itens avaliados no Check-list

* check-list de acordo com as RDC 216/2004 e 275/2002

Tabela 2- Verificação de conformidades e não conformidades das condições físicas e higiênico-sanitárias das cozinhas e dos manipuladores encontradas na escola "B" após aplicação de check-list**

\section{Escola B}

$\mathrm{N}^{*} \quad \%$

Conformidades $18 \quad 72$

Não conformidades $7 \quad 28$

\section{Total 25100 \\ $N^{*}$ - Numero de itens avaliados no Check-list \\ * check-list de acordo com as RDC 216/2004 e 275/2002}

Tabela 3- Verificação de conformidades e não conformidades das condições físicas e higiênico-sanitárias das cozinhas e dos manipuladores encontradas na escola "C" após aplicação de check-list** 


\section{Escola C \\ $\mathrm{N}^{*} \quad \%$}

Conformidades

$16 \quad 64$

Não conformidades

936

\begin{tabular}{lll}
\hline Total & 25 & 100
\end{tabular}

$N^{*}$ - Numero de itens avaliados no Check-list

* check-list de acordo com as RDC 216/2004 e 275/2002

Os resultados do check-list revelam que os percentuais de conformidades encontradas neste estudo nas três escolas são maiores que os percentuais de não conformidades. Ou seja, a avaliação das condições físicas e higiênico-sanitárias das cozinhas e dos manipuladores de alimentos das escolas no presente estudo mostrou-se satisfatória, apesar da constatação de algumas não-conformidades. Tal resultado pode ser entendido como um indício da aplicação das RDCs vigentes no país.

Outros estudos também apresentam percentuais semelhantes de conformidades em relação às condições físicas e higiênico-sanitárias das cozinhas e dos manipuladores de alimentos. Mariano \& Moura (2008) obtiveram em sua pesquisa no interior de São Paulo, um percentual de $67,4 \%$ de conformidades em uma unidade produtora de refeições. Em estudo semelhante desenvolvido em uma unidade de alimentação por Souza et al., (2009) em Timóteo-MG, verificou-se um percentual de $76 \%$ de conformidade no estabelecimento pesquisado.

Tabela 4- Resultados das não conformidades das condições físicas e higiênico sanitárias encontradas nas escolas públicas de Rianápolis-GO.

\begin{tabular}{|c|c|c|c|c|c|c|}
\hline \multirow{3}{*}{ CARACTERÍSTICAS } & \multicolumn{6}{|c|}{ AVALIAÇÃO DAS NÃO CONFORMIDAS DAS ESCOLAS } \\
\hline & \multicolumn{2}{|c|}{ A } & \multicolumn{2}{|c|}{ B } & \multicolumn{2}{|c|}{$\mathbf{C}$} \\
\hline & $\mathbf{N}$ & $\%$ & $\mathbf{N}$ & $\%$ & $\mathbf{N}$ & $\%$ \\
\hline LIXEIRAS SEM PEDAL & 1 & 4 & 0 & 0 & 1 & 4 \\
\hline SAPATO ABERTO & 1 & 4 & 1 & 4 & 1 & 4 \\
\hline
\end{tabular}




\begin{tabular}{lcccccc}
\hline VENTILAÇÃO INADEQUADA & 0 & 0 & 0 & 0 & 1 & 4 \\
INCAPACITAÇÃO EM BPF & 0 & 0 & 0 & 0 & 1 & 4 \\
$\begin{array}{l}\text { TEMPERATURA DO AMBIENTE } \\
\text { INADEQUADA }\end{array}$ & 1 & 4 & 1 & 4 & 1 & 4 \\
$\begin{array}{l}\text { NÃO REGISTRO TEMPERATURA } \\
\text { DOS REFRIGERADORES }\end{array}$ & 1 & 4 & 1 & 4 & 1 & 4 \\
$\begin{array}{l}\text { PRODUTOS DE LIMPEZA } \\
\text { INADEQUADOS }\end{array}$ & 1 & 4 & 1 & 4 & 1 & 4 \\
$\begin{array}{l}\text { LUMINÁRIAS SEM PROTETORES } \\
\text { PAREDE SEM REVESTIMENTO }\end{array}$ & 1 & 4 & 1 & 4 & 1 & 4 \\
E RACHADURAS & 1 & 4 & 1 & 4 & 1 & 4 \\
SECAGEM DE MÃO INADEQUADA & 1 & 4 & 1 & 4 & 1 & 4 \\
& & & & & & \\
\hline
\end{tabular}

Os resultados referentes às não conformidades das condições físicas e higiênicosanitárias encontradas nas cozinhas das três escolas pesquisadas neste estudo foram listadas na Tabela 4. As não conformidades encontradas nas três escolas pesquisadas foram: "sapatos abertos", "não registro de temperatura dos refrigeradores e do ambiente", "produtos de limpeza inadequados", "luminárias sem protetores", "paredes sem revestimentos e com rachaduras". No entanto, as não conformidades consideradas "mais críticas" encontradas no estudo foram: "secagem de mão inadequada", "lixeiras sem pedal", "temperatura do ambiente inadequada", "não registro da temperatura dos refrigeradores" e "falta de treinamento".

As escolas A e B se encontraram irregulares quanto à "secagem de mão inadequada" uma vez que as mesmas utilizavam detergente comum para lavagem das mãos e uma única toalha de tecido para secagem das mãos. Em seus estudos em Porto Velho-RO, Aguiar et al (2011) encontraram também esta inadequação nas cozinhas de duas das três lanchonetes pesquisadas. $\mathrm{O}$ uso da toalha de tecido por todas as manipuladoras apresenta um meio propício de proliferação de micro-organismos e parasitas, sendo uma fonte de contaminação para as mãos e posteriormente para os alimentos. Segundo as RDCs vigentes, o processo de lavagem das mãos deve ser feito em local adequado e de maneira correta, utilizando papel toalha não reciclável ou ar quente para secar as mãos e sabonete líquido 
antisséptico e inodoro, desta forma o resultado final para manipulação dos alimentos será satisfatório (SOUZA, 2004).

Outra não-conformidade relatada no estudo foi a utilização de "lixeiras sem pedal" expostas no ambiente de manipulação dos alimentos pelas escolas A e C. As inadequação quanto às lixeiras também foram relatadas por Cardoso et al (2010) ao pesquisar cozinhas de escolas em Salvador-BA. Segundo as normas das RDCs, as lixeiras devem ser devidamente tampadas e contar com o auxílio de pedal, pois as mesmas são focos de contaminação cruzada atraindo insetos e roedores que provocam doenças. As lixeiras devem ser mantidas em condições adequadas de higiene e conservação e a cada esvaziamento deve ser feito o processo de limpeza (SANTOS JUNIOR, 2008).

Quanto às não conformidades "temperatura do ambiente inadequada" e "não registro da temperatura dos refrigeradores", verificou-se que nas cozinhas das três escolas a temperatura tanto do ambiente quanto a das geladeiras e dos freezers que são armazenados os alimentos estavam inadequadas ou insuficientes para demanda dos mesmos. Como afirma BRASIL (2004), os micro-organismos podem se multiplicar rapidamente nos alimentos em temperaturas entre $5^{\circ} \mathrm{C}$ a $60^{\circ} \mathrm{C}$. Por isso é muito importante que a geladeira e o freezer estejam em perfeitas condições de funcionamento e na temperatura adequada que é abaixo de $5^{\circ} \mathrm{C}$. Vale ressaltar que os alimentos produzidos nos ambientes em que a temperatura não estiver dentro dos parâmetros estabelecidos pelas RDCs vigentes devem ser desprezados (SÃO PAULO, 2003)

Embora os resultados obtidos nas escolas pesquisadas tenham evidenciado que as condições higiênico-sanitárias das cozinhas e dos manipuladores de alimentos foram satisfatórias, foi possível detectar itens a serem melhorados tais como aqueles verificados nas não-conformidades. Portanto, ressalta-se aqui a importância das boas práticas em serviços de alimentação e a elaboração e implantação de um Manual de Boas Práticas nas escolas, visando garantir a qualidade e a conformidade dos alimentos com a legislação sanitária, proporcionando segurança alimentar aos alunos.

\section{CONCLUSÃO}

Neste estudo foram avaliados 15 manipuladores de alimentos de três escolas da rede pública de Rianápolis-GO através da aplicação de um check-list que avaliou as condições físicas e higiênico-sanitárias das cozinhas e dos manipuladores de alimentos. A escola A, 
apresentou 17 (68\%) de conformidade e 8 (32\%) de não conformidades; a escola B, 18 (72\%) conformidade e 7 (28\%) de não conformidades e a escola C, 16 (64\%) de conformidade e 9 $(36 \%)$ de não conformidades. Os resultados do check-list revelam que os percentuais de conformidades encontradas neste estudo nas três escolas são maiores que os percentuais de não conformidades. Ou seja, a avaliação das condições físicas e higiênico-sanitárias das cozinhas e dos manipuladores de alimentos das escolas no presente estudo mostrou-se satisfatória, apesar da constatação de algumas não-conformidades. Tal resultado pode ser entendido como um indício da aplicação das RDCs vigentes no país.

Quanto às não conformidades em relação às condições físicas e higiênico-sanitárias encontradas nas cozinhas das três escolas pesquisadas neste estudo verificou-se que as mais frequentes foram: "sapatos abertos", "não registro de temperatura dos refrigeradores e do ambiente", "produtos de limpeza inadequados", "luminárias sem protetores", “paredes sem revestimentos e com rachaduras". No entanto, as não conformidades consideradas "mais críticas" encontradas no estudo foram: "secagem de mão inadequada", "lixeiras sem pedal", "temperatura do ambiente inadequada", "não registro da temperatura dos refrigeradores" e "falta de treinamento". Segundo as RDCs vigentes, todos estes parâmetros devem ser observados e evitados para garantir o preparo de alimentos seguros.

\section{REFERÊNCIAS}

AGUIAR, A. M. M.; BATISTA, B. V. A.; SANTOS, I. H. V. S.; OLIVEIRA, L. M. L. Avaliação da eficácia de uma intervenção sobre as boas práticas de higiene em três lanchonetes de uma escola particular em Porto Velho- RO. Saber Científico, Porto Velho, 3 (1): 70-90, jul/dez., 2011.

AKUTSU, R.C.; BOTELHO, R.A.; CAMARGO, E.B.; SÁVIO, K.E.O.; ARAÚJO, W.C. Adequação de boas práticas de fabricação em serviços de alimentação. Revista de Nutrição, Campinas, v.18, n.3, p.419-27, 2005.

ALMEIDA, F. H.; SOUZA, E. C. G. Alimentação escolar: as instituições de ensino do município de Muriaé e suas intervenções. Muriaé: Faculdade de Minas, 2007. 
BRASIL. Ministério da Saúde. Agência Nacional de Vigilância Sanitária- ANVISA. Cartilha sobre Boas Práticas para serviços de alimentação Resolução-RDC nº 216/2004. Brasília. 2004

BRASIL. Ministério da Saúde. Secretaria de Vigilância em Saúde. Vigilância

Epidemiológica das Doenças de Transmissão Hídrica e Alimentar -VEDTHA. Brasil, 2011. Disponível em:

〈http://portal.saude.gov.br/portal/arquivos/pdf/10_passos_para_investigacao_surtos.pdf $>$ Acesso em: 23 de outubro 2012.

BRASIL. Ministério da Saúde. Secretaria de Vigilância em Saúde. Vigilância epidemiológica das doenças transmitidas por alimentos no Brasil, 1999 - 2004. Boletim eletrônico Epidemiológico, n.6, 2005. Disponível em: $<$ http://portal.saude.gov.br/portal/arquivos/pdf/ano05_n06_ve_dta_brasil.pdf $>$ Acesso em: 19 de Setembro 2012.

CARDOSO, R. C. V.; GÓES, J. Â. W.; ALMEIDA, R. C. C.; GUIMARÃES, A. G.; BARRETO, D. L.; DA SILVA, S. A.; FIGUEIREDO, K. V. N. A.; VIDAL Jr, P. O.; SILVA, E. O.; HUTTNER, L. B. Programa Nacional de Alimentação Escolar: Há Segurança na Produção de alimentos em Escolas de Salvador (Bahia)? Revista de Nutrição. v.23, n.5, p.801-811, Set/Out, 2010.

DA SILVA, B. C.; FORTUNA, J. L. Condições higiênico-sanitárias na manipulação de alimentos, em cozinhas e cantinas de escolas públicas municipais de Mucuri,BA. Revista Higiene Alimentar, São Paulo, v.25, n.202/203, p.51-56, novembro/dezembro, 2011.

FUNDO NACIONAL DE DESENVOLVIMENTO DA EDUCAÇÃO (FNDE). Resolução n 32 de 10 de agosto de 2006. Estabelece normas para a execução do Programa Nacional de Alimentação Escolar - PNAE, [Brasília], 2006.

MARIANO, C. G.; MOURA, P. N. Avaliação das boas práticas de fabricação em unidade produtora de refeições (UPR) auto-gestão do interior do estado de São Paulo. Rev. Salus, v. 2, n. 2, p. 73-81, jul./dez. 2008 
MELLO, A. G. Condições higiênico-sanitárias na produção de refeições em restaurantes públicos populares localizados no Estado do Rio de Janeiro. 2009. 130 f. Dissertação (Mestrado em Vigilância Sanitária). Fundação Oswaldo Cruz, Rio de Janeiro, 2009.

PANIZZA, F.; LEMES, G. A. S.; BARNABÉ, A. S.; POPOLIM, W. D. Percepção do nutricionista frente a notificações de surtos alimentares. Revista Higiene Alimentar, São Paulo, v.25, n.202/203, p.22-28, novembro/dezembro, 2011.

ROUGEMONT, A. J. Alimentos seguros - necessidade ou barreira comercial? Perspectivas Online, Campos dos Goytacazes, v. 1 nº 2 p.62-70, 2007.

SÃO PAULO. Secretaria Municipal da Saúde. Critérios e parâmetros para a produção de alimentos e bebidas, aplicados às empresas de alimentos. Portaria 2.535/03. Diário Oficial do Município de São Paulo 2003; 24 out.

SOUZA, C. H.; SATHLER. J.; JORGE, M. N.; HORST, R. F. M. L. Avaliação das Condições Higiênico-Sanitárias em uma Unidade de Alimentação e Nutrição Hoteleira, na cidade de Timóteo- MG. NUTRIR GERAIS - Revista Digital de Nutrição, Ipatinga, fev./jul. 2009, v. 3, n. 4, p. 312-329.

SOUZA, S. S. Alimentos Seguros: Orientações técnicas. São Paulo, Prefeitura do Município de São Paulo. Secretaria Municipal da Saúde Coordenação de Vigilância em Saúde, Gerência de Comunicação e Educação, 2004.

\section{APÊNDICE 1}

\begin{tabular}{|c|c|c|}
\hline CHECK LIST & C & NC \\
\hline $\begin{array}{l}\text { A aparência dos manipuladores de alimentos está de } \\
\text { acordo com as normas descritas pela vigilância sanitária } \\
\text { (Usa uniforme limpos, não usam adornos, os sapatos são }\end{array}$ & & \\
\hline
\end{tabular}




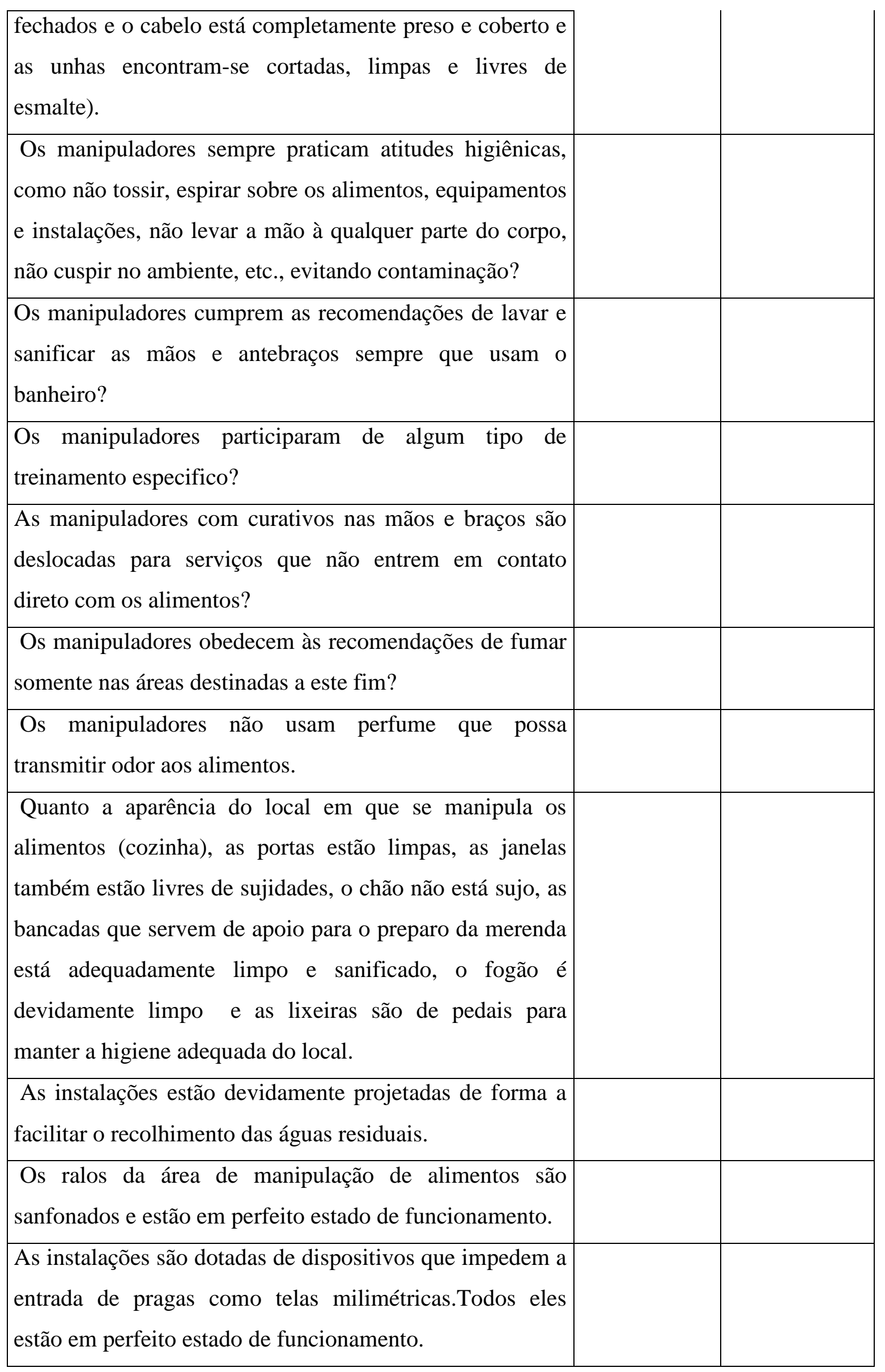




\begin{tabular}{|c|c|}
\hline \multicolumn{2}{|l|}{$\begin{array}{l}\text { A temperatura está sendo aferida em todas as áreas em } \\
\text { que seu controle é fundamental. }\end{array}$} \\
\hline $\begin{array}{l}\text { A ventilação é adequada ao controle de odores } \\
\text { indesejáveis e vapores que podem alterar os produtos ou } \\
\text { mascarar odores de deterioração ou de alguma forma } \\
\text { alterar o produto. }\end{array}$ & \\
\hline $\begin{array}{l}\text { Sistema de esgoto (recolhimento das águas) é capaz de } \\
\text { drenar todo o volume produzido. }\end{array}$ & \\
\hline $\begin{array}{l}\text { A utilização de produtos de higienização nos } \\
\text { (equipamentos, utensílios e ambiente) está de acordo com } \\
\text { o preconizado pelo Ministério da Saúde (Desinfectante e } \\
\text { Sanitizante). }\end{array}$ & \\
\hline $\begin{array}{l}\text { A iluminação existente é adequada para a atividade } \\
\text { desenvolvida no local. }\end{array}$ & \\
\hline Todas as luminárias possuem protetores. & \\
\hline Todas as lâmpadas estão funcionando perfeitamente. & \\
\hline $\begin{array}{l}\text { O sistema de abastecimento de água é ligado à rede } \\
\text { pública. }\end{array}$ & \\
\hline $\begin{array}{l}\text { As paredes das escolas estão livres de sujeiras, rachaduras } \\
\text { e possuem revestimento de azulejos em todas as paredes } \\
\text { até o teto? }\end{array}$ & \\
\hline $\begin{array}{l}\text { No armazenamento dos alimentos, há presença de } \\
\text { alimentos encostados na parade, no chão e dentro de } \\
\text { caixas de papelão? }\end{array}$ & \\
\hline $\begin{array}{l}\text { Os alimentos estão separados dos materias de limpeza e } \\
\text { sanificantes? }\end{array}$ & \\
\hline $\begin{array}{l}\text { As geladeiras e os frezzers utilizados para o } \\
\text { armazenamento de frios estão em bom estado de } \\
\text { conservação e limpos? }\end{array}$ & \\
\hline $\begin{array}{l}\text { Há registros contínuos dos controles de temperatura } \\
\text { (Geladeiras e Freezer). }\end{array}$ & \\
\hline
\end{tabular}


Instalações sanitárias dotadas de produtos destinados à higiene pessoal: papel higiênico, sabonete líquido inodoro antisséptico, toalhas de papel não recicladas para as mãos ou outro sistema higiênico e seguro para secagem . 